\title{
Left atrial appendage closure for stroke risk reduction in patients with non-valvular atrial fibrillation and high bleeding risk-The Watchman Device
}

\author{
Menfil Orellana-Barrios MD, Mohammad M. Ansari MD
}

Stroke is a well-established and devastating thromboembolic complication of atrial fibrillation. ${ }^{1}$ The risk of stroke in patients with non-valvular AF has been well studied, and stroke risk reduction is a major goal of therapy. ${ }^{2}$ Anticoagulation is a well-established method to reduce the incidence of stroke in patients with AF but is underused and accompanied with a well-known risk of hemorrhagic complications. ${ }^{3}$ To quantify the risk of stroke in AF, several clinical tools have been published. The most widely used are the CHADS2 and CHA2DS2VASc scores, which use clinical patient characteristics to predict the annual risk for stroke in percent risk per year (Figure 1)., ${ }^{4,5}$ In addition, several scoring systems, such as the ATRIA, ORBIT, ABC and HAS-BLED scores, have been published to evaluate the risk of bleeding while on anticoagulation. ${ }^{6}$ These also use clinical characteristics to predict the annual rate of major bleeding (Table 1). ${ }^{7}$ Of these, the HAS-BLED score has been endorsed as a method of quantifying the risk of bleeding by US guidelines. The risk of major bleeding within 1 year increases progressively with accumulative HASBLED points (bleeds per 100 patient-years): 0 (1.13), 1 (1.02), 2 (1.88), 3 (3.74), 4 (8.7), 5 (12.5). The predictive value of scoring systems for patients using direct oral anticoagulants is currently under study. Current US guidelines recommend anticoagulation of patients with a CHADS2VASC score of 2 or above, while European guidelines do permit anticoagulation (or aspirin) with scores of 1 or above..$^{2,8}$

Despite the very useful and simple to use clinical scoring systems described above, clinicians not infrequently encounter patients who have both a

Corresponding author: Mohammad M. Ansari Contact Information: Mac.ansari@ttuhsc.edu DOI: 10.12746/swrccc.v8i33.631 high thromboembolic risk and a high bleeding risk. This scenario poses a peculiar clinical conundrum. In these patients, there is distinct clinical need for an intervention that will reduce stroke risk while minimizing potential bleeding. Approximately $91 \%$ of left atrial thrombi arise for the atrial appendage (LAA), making this area an attractive therapeutic target for stroke risk reduction. ${ }^{10}$ Although surgical excision of the LAA is possible, this technique is not commonly used unless the patient undergoes cardiothoracic surgery for another indication. ${ }^{2}$

Percutaneous appendage closure is a relatively novel concept and technique in which a left atrial appendage occluder (LAAO) is implanted at the ostia of the LAA to isolate it from the rest of the atrium. The Watchman device (Boston Scientific, Natick, MA, USA) is a LAAO, engineered to have a parachute-shaped self-expanding nitinol frame structure covered with a permeable polyester membrane (polyethylene terephthalate [PET]). As seen in Figure 2, the Watchman device has 10 perimeter active fixation barbs which aid in providing fixation and stability within the LAA. It is delivered via a $14 \mathrm{~F}$ transseptal $75-\mathrm{cm}$ sheath (inner

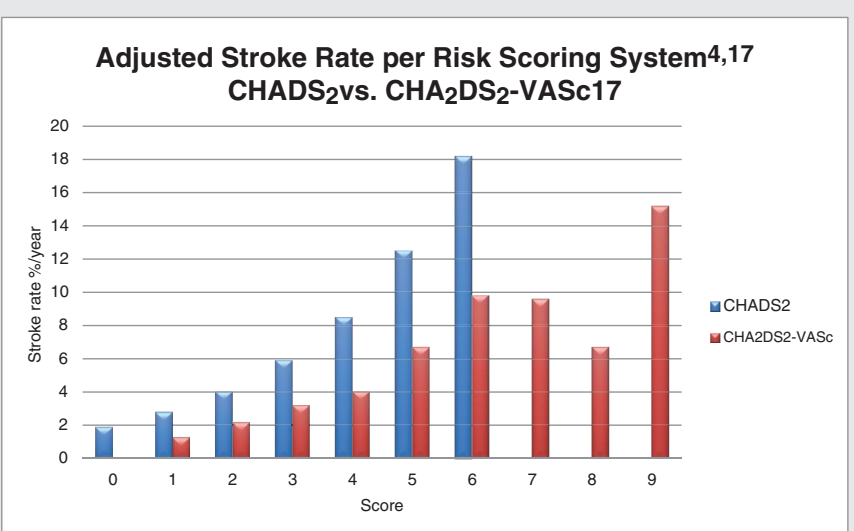

Figure 1. Stroke rate per CHADS2 and CHA2DS2VASc Category. ${ }^{4,17}$ 
Table 1. HAS-BLED Clinical Score ${ }^{7}$

\begin{tabular}{|l|l|}
\hline Risk factor & Score \\
\hline Hypertension & 1 \\
\hline Abnormal Renal/Liver function & 1 for each \\
\hline Stroke & 1 \\
\hline Bleeding & 1 \\
\hline Labile INRs & 1 \\
\hline Elderly $>65$ years & 1 \\
\hline Drugs/Alcohol & 1 for each \\
\hline
\end{tabular}

diameter $12 \mathrm{~F})$. There are 5 available sizes $(21,24$, 27,30 , and $33 \mathrm{~mm}$ ) to accommodate to the maximum LAA ostium diameter (usually oversized to $10-20 \%$ of the measured diameter). The device is designed to be partially recaptured and redeployed if location or size are unsatisfactory at the time of implantation. ${ }^{11}$ The first clinical experience with the Watchman device was published in $2002 .{ }^{11}$ Since then, two randomized clinical trials have been published.

The PROTECT-AF trial (2014) is a multicenter (59 hospitals, 4998 screened patients), randomized (2:1), unblinded Bayesian design study including 707 subjects ( $90 \%$ white) with non-valvular AF, and a CHADS2 score $\geq 1 .{ }^{12}$ Patients were randomized to LAAO or warfarin (INR 2-3). The protocol predetermined that after implantation, patients would receive ASA (81-325 $\mathrm{mg}$ ) and warfarin for 45 days and have TEE evaluations at 45 days, 6 months, and 12 months. At 45 days, patients with minimal peri-device flow and no visible clot were switched to ASA and clopidogrel $75 \mathrm{mg}$ daily. At the 6-month mark, clopidogrel was discontinued, and patients remained only on ASA.

Outcomes included a composite of stroke, systemic embolism, and cardiovascular/unexplained death. The mean follow-up was $3.8+1.7( \pm S D)$ years. The mean CHADS2 score was 2.2 points. The event rates were $8.4 \%$ and $13.9 \%$ in the device and warfarin groups, respectively (rate ratio $0.60 ; 95 \%$ confidence interval, $0.41-1.05)$, meeting prespecified criteria for non-inferiority and superiority. Additionally, patients in the device group had lower rates of cardiovascular $(3.7 \%$ vs. 9.0\%; hazard ratio $0.40, \mathrm{Cl} 0.21-0.75$ ) and all-cause mortality $(12.3 \%$ vs. $18 \%$; hazard ratio 0.66 , $\mathrm{Cl} 0.45-$ 0.98 ) in comparison to warfarin. Most of the beneficial outcomes was driven by lower rates of hemorrhagic stroke and cardiovascular death. In this trial, the rate of intracranial hemorrhage in the warfarin group was higher when compared to other trials $(1.1 \%$ /year versus $0.4-0.5 \% / y e a r)$. The ischemic stroke rate was similar between both groups (device: $1.4 \% /$ year vs. warfarin $1.1 \% /$ year; $P=0.49$ ).

Concerns related to PROTECT-AF trial included the inclusion of patients with CHADS2 scores of 1 (who would not require anticoagulation per US guidelines) and acute safety events, in particular a high initial accumulation of procedure related complications in the device arm (6 procedure related stroke in 463 patients). Given these limitations, at the request of the FDA, the PREVAIL study was designed and conducted.

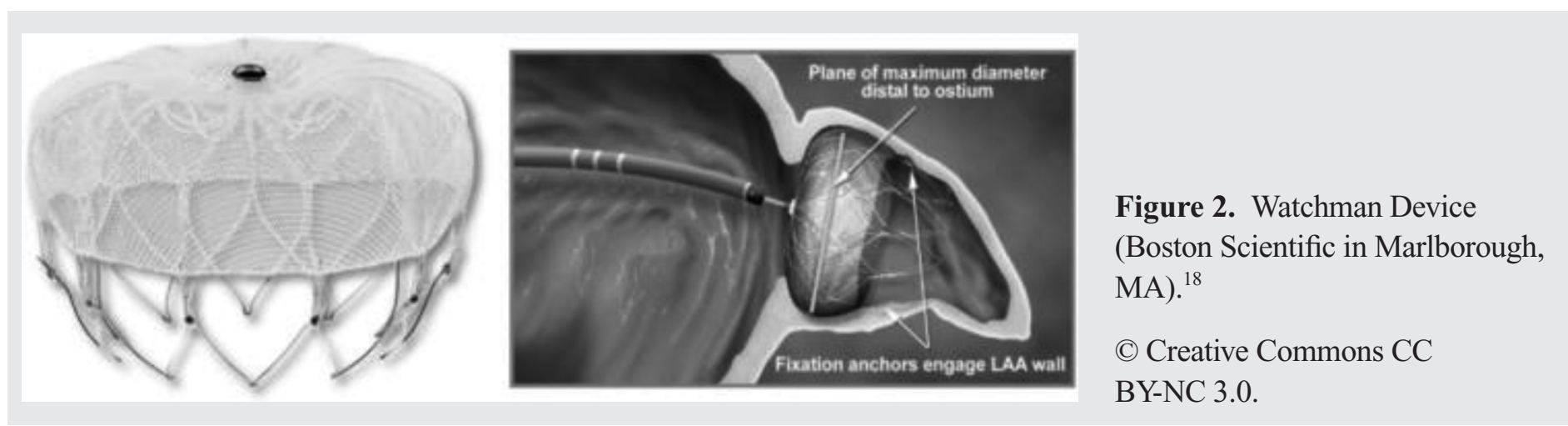


The PREVAIL trial (2014) is a multicenter (50 US sites) randomized trial designed to assess the safety and efficacy of the Watchman device for stroke prevention in patient with non-valvular AF versus patient with long-term warfarin therapy. ${ }^{13}$ The study included 475 patients (269 in device arm) with CHADS2 score $\geq 2$ or 1 and another predetermined clinical criterion (female age $\geq 75$, LVEF $\geq 30$ and $<35 \%$, age $65-74$, $\mathrm{DM}$ or $\mathrm{CAD}$, and age $\geq 65$ with $\mathrm{CHF}$ ). The co-primary endpoints were 1) composite of hemorrhagic/ischemic stroke, systemic embolism, and cardiovascular/unexplained death; 2) composite of ischemic stroke or systemic embolism excluding first 7 days after randomization; and 3) composite of all-cause death, ischemic stroke, systemic embolism or device related event requiring surgery or major endovascular intervention.

The study protocol indicated that patients with device implants would continue on ASA $81 \mathrm{mg}$ daily and warfarin (INR 2-3) for 45 days and then have TEE evaluation. If LAA was sealed ( $<5 \mathrm{~mm}$ peri-device flow and no thrombus on device), then therapy was transitioned to ASA 81-325 mg daily with clopidogrel $75 \mathrm{mg}$ for the next 6 months. If at 45 days the LAA seal was inadequate, then warfarin was continued until the seal was achieved and at this point, patients were switched to ASA 81-325 mg daily. Follow-up visits were at 45 days, 6 months, 9 months, and then biannually.

At 18 months, the first primary efficacy endpoints were similar in both arms but statistical non-inferiority was not achieved (device rate 0.0253 , warfarin rate 0.0200 ; rate difference $0.0053 ; \mathrm{Cl}-0.019$ to 0.0276 ). The second predefined outcome regarding lateischemic efficacy had a 0.0253 rate for the device group versus 0.0200 in the warfarin arm (risk difference $0.0053, \mathrm{Cl}-0.0190$ to 0.0273 ). Non-inferiority criteria were achieved for this endpoint. The third endpoint was evaluated only in the device arm, and success was achieved as the percentage of patient meeting the endpoint (2.2\%) was statistically less than the performance goal of $2.67 \%$ (one sided $95 \% \mathrm{Cl}$ of $2.65 \%$ ). Procedural success in PREVAIL was $95.1 \%$, compared to $90.9 \%$ in PROTECT AF. The 7-day postprocedure complication rate significantly decreased from $8.7 \%$ in the PROTECT AF trial to $4.2 \%$ in the PREVAIL trial. Procedural and device related strokes decreased from $1.1 \%$ in PROTECT AF trial to $0.4 \%$ in PREVAIL trial $(p=0.007)$.

An important detail in the PREVAIL trial is that the warfarin arm over-performed in relation to the expected ischemic stroke event rate, which was $0.71 / 100$ patient years, compared to rates ranging from 1.6-2.20 in the large DOAC trials versus warfarin (ARISTOTLE, ROCKET-AF, RE-LY). ${ }^{13}$ It is also important to note that the safety and efficacy of Watchman versus DOACs have not been established.

Data acquired using the PROTECT AF trial cohort in addition to nonrandomized continued access protocol registry have shown a significant decline in the rate of procedure or device related safety events within 7 days for the procedure. Although nonrandomized, these data suggest that there is a significant improvement in the safety of LAAO with Watchman with increased operatory experience. ${ }^{14}$

A published meta-analysis including 5-year data using the PREVAIL and PROTECT AF cohorts reported statistically similar all stroke/systemic embolization rates between the device and warfarin arms, although the stroke rate in the device arm was numerically higher. However, differences in hemorrhagic stroke (HR: $0.20 ; p=0.0022$ ), disabling/fatal stroke $(0.45 ; p=0.03)$, cardiovascular/unexplained death (HR: 0.59; $p=0.027$ ), all-cause death (HR: 0.73; $p=0.035$ ), and post-procedure bleeding (HR: 0.48; $p=0.0003$ ) were significantly better in the device arm. ${ }^{15}$

A new generation Watchman FLX (Boston Scientific, Marlborough, MA, USA) was made available in Europe in 2015. It had a few upgrades, including a new sizing scheme $(20,24,27,31,35 \mathrm{~mm})$, reduced length, flat proximal face, $80 \%$ more LAA contact points, atraumatic closed distal end with fluoroscopic marker, $12 \mathrm{~J}$-shaped anchors in 2 rows, and increased compression range (10-27\%). The manufacturing company withdrew this device from market due to higher than anticipated embolization rates (3.8\% of 209 implants). Another design upgrade is expected in $2018 .^{16}$

LAAO is not a universal substitution for anticoagulation in reduction of stroke risk in non-valvular AF. Particularly in an era in which the DOACS have 
demonstrated reduced composite stroke and systemic embolic events and statistically significant reduction in all-cause mortality, with similar ischemic stroke prevention rates compared to warfarin, future studies involving LAAO and DOACs will be important. In the meantime, carefully selected patients may be given an option with which they can reduce their thromboembolic risk significantly with concomitant limitation of the bleeding complications. Although there is a small signal of increased peri-procedural morbidity, many patients and clinicians may find an acceptable answer to the always difficult decision of anticoagulation of AF patients with both increased thromboembolic and bleeding risk. It is in this type of scenario where the maxim of "individualized decision-making" is of utmost importance.

\section{Keywords: atrial appendage, atrial clot, Watchman device}

Article citation: Orellana-Barrios M, Ansari MA. Left atrial appendage closure for stroke risk reduction in patients with non-valvular atrial fibrillation and high bleeding riskThe Watchman Device. The Southwest Respiratory and Critical Care Chronicles 2020;8(33):35-39

From: Department of Internal Medicine, Texas Tech University Health Sciences Center, Lubbock, Texas

Submitted: 9/23/2019

Accepted: $12 / 28 / 2019$

Reviewer: Scott Shurmur MD

Conflicts of interest: none

This work is licensed under a Creative Commons

Attribution-ShareAlike 4.0 International License.

\section{REFERENCES}

1. Violi F, Pastori D, Pignatelli P. Mechanisms and management of thrombo-embolism in atrial fibrillation. J Atr Fibrillation $2014 ; 7$.

2. January CT, Wann LS, Alpert JS, et al. AHA/ACC/HRS guideline for the management of patients with atrial fibrillation: a report of the American College of Cardiology/American Heart Association Task Force on Practice Guidelines and the Heart Rhythm Society. J Am Coll Cardiol 2014;64:e1-76.
3. Sterne JA, Bodalia PN, Bryden PA, et al. Oral anticoagulants for primary prevention, treatment and secondary prevention of venous thromboembolic disease, and for prevention of stroke in atrial fibrillation: systematic review, network meta-analysis and cost-effectiveness analysis. Health Technol Assess 2017;21:1-386.

4. Gage BF, Waterman AD, Shannon W, et al. Validation of clinical classification schemes for predicting stroke: results from the National Registry of Atrial Fibrillation. JAMA 2001; 285:2864-2870.

5. Lip GY, Nieuwlaat R, Pisters R, et al. Refining clinical risk stratification for predicting stroke and thromboembolism in atrial fibrillation using a novel risk factor-based approach: the euro heart survey on atrial fibrillation. Chest 2010;137:263-272.

6. Dzeshka MS, Lane DA, Lip GY. Stroke and bleeding risk in atrial fibrillation: navigating the alphabet soup of risk-score acronyms (CHADS2, CHA2 DS2 -VASc, R2 CHADS2, HASBLED, ATRIA, and more). Clin Cardiol 2014;37:634-644.

7. Pisters R, Lane DA, Nieuwlaat R, et al. A novel user-friendly score (HAS-BLED) to assess 1-year risk of major bleeding in patients with atrial fibrillation: the Euro Heart Survey. Chest 2010;138:1093-1100.

8. Kirchhof P, Benussi S, Kotecha D, et al. Guidelines for the management of atrial fibrillation developed in collaboration with EACTS. Eur Heart J 2016;37:2893-2962.

9. Lip GY. Implications of the CHA2DS2-VASc and HASBLED Scores for thromboprophylaxis in atrial fibrillation. Am J Med 2011;124:111-114.

10. Blackshear JL, Odell JA. Appendage obliteration to reduce stroke in cardiac surgical patients with atrial fibrillation. The Annals Thoracic Surgery 1996;61:755-759.

11. Sick PB, Schuler G, Hauptmann KE, et al. Initial worldwide experience with the WATCHMAN left atrial appendage system for stroke prevention in atrial fibrillation. J Am Coll Cardiol 2007;49:1490-1495.

12. Reddy VY, Sievert H, Halperin J, et al. Percutaneous left atrial appendage closure vs warfarin for atrial fibrillation: a randomized clinical trial. JAMA 2014;312:1988-1998.

13. Belgaid DR, Khan Z, Zaidi M, et al. Prospective randomized evaluation of the watchman left atrial appendage closure device in patients with atrial fibrillation versus long-term warfarin therapy: The PREVAIL trial. Int J Cardiol 2016;219:177-179.

14. Reddy VY, Holmes D, Doshi SK, et al. Safety of percutaneous left atrial appendage closure: results from the Watchman Left Atrial Appendage System for Embolic Protection in Patients with AF (PROTECT AF) clinical trial and the Continued Access Registry. Circulation 2011;123:417-424.

15. Reddy VY, Doshi SK, Kar S, et al. 5- year outcomes after left atrial appendage closure: from the PREVAIL and PROTECT AF Trials. J Am Coll Cardiol 2017;70:2964-2975. 
16. Grygier M, Olasin ska-Wis niewska A, Araszkiewicz A, et al. The Watchman FLX-a new device for left atrial appendage occlusion-design, potential benefits and first clinical experience Postepy Kardiol Interwencyjnej 2017:62-66.

17. Lip GY, Frison L, Halperin JL, et al. Identifying patients at high risk for stroke despite anticoagulation: a comparison of contemporary stroke risk stratification schemes in an anticoagulated atrial fibrillation cohort. Stroke. 2010 Dec;41(12): 2731-8. Epub 2010 Oct 21.

18. Akinapelli A, Bansal O, Chen JP, et al. Left Atrial Appendage Closure-The WATCHMAN Device. Curr Cardiol Rev. 2015;11(4):334-340. 